\title{
Creatinina sérica elevada en el paciente hipotiroideo: miopatía o verdadera nefropatía? Propuesta de clasificación.
}

\author{
(Elevated Serum Creatinine in Hypothyroidism: \\ Myopathy or Real Nephropathy? Classification Proposal)
}

\author{
Carmen Lidia Guerrero-Lobo
}

\section{Resumen:}

Justificación: En el paciente hipotiroideo la creatinina sérica puede aumentar no solo por fallo renal, sino también por miopatía en presencia de función renal normal. Se reportan 2 pacientes representativos de sendos tipos de la clasificación aquí propuesta sobre la asociación disfunción tiroidea / creatinina sérica. El primer enfermo presenta aumento de la creatinina sérica secundario a miopatía hipotiroidea con función renal normal (tipo 1), esta se puede complicar con rabdomiolisis y causar insuficiencia renal aguda en hipotiroideos no diagnosticada. El otro paciente con creatina sérica alta mejoró su función renal al tratarse su hipotiroidismo (tipo 2). Ocasionalmente, este ha permanecido oculto y al ser diagnosticado y tratado, se mejora la función renal. El hipotiroidismo cursa con hipercolesterolemia y si no se valora la función tiroidea y se administran estatinas, se empeora el daño muscular del paciente. Lo mismo ha sucedido con el uso de gemfibrozil en condiciones similares. El tercer tipo de daño renal relacionado con hipotiroidismo es la proteinuria en rango de síndrome nefrótico, vinculada con suspensión de la levotiroxina. Las biopsias muestran lesiones histológicas de diversas glomerulopatías, que mejoran con la administración de levotiroxina. En pacientes con tiroiditis de Hashimoto aparecen otras glomerulopatías que responden a los glucocorticoides.

Conclusión: 1) La hormona estimulante de la tiroides (TSH por sus siglas en inglés) debe cuantificarse en pacientes

Universidad de Costa Rica, Facultad de Medicina, Escuela de Medicina, Departamento de Farmacología Clínica y Toxicología

Abreviaturas: CK, creatinina fosfocinasa; $\mathrm{CrS}$ : creatinina sérica; $\mathrm{FG}$, filtración glomerular; ICC, insuficiencia cardíaca congestiva;IRA, insuficiencia renal aguda; IRC, insuficiencia renal crónica, LT4, levotiroxina; T4, hormona tiroidea; TSH, hormona estimulante de la tiroides; NU, nitrógeno ureico. Correspondencia: Carmen Lidia Guerrero-Lobo. E-mail: Iguerrer@ice.co.cr. Apartado postal: $4044-1000$

ISSN 0001-6002/2008/51/1/44-48 Acta Médica Costarricense, (C2009 Colegio de Médicos y Cirujanos con creatina sérica aumentada, en aquellos con colesterol elevado sin una causa evidente y en los que tienen polimiositis o rabdomiolisis con creatina fosfocinasa elevada e insuficiencia renal aguda. 2) Se debe solicitar creatina sérica a los pacientes hipotiroideos que usan amiodarona y a quienes suspenden el tratamiento con levotiroxina, pues pueden presentar disminución de su función renal. 3) Un paciente hipotiroideo que descontinuó la levotiroxina puede desarrollar un síndrome nefrótico que puede responder al tratamiento con glucocorticoides o levotiroxina, si su origen es inmunológico o no.

Descriptores: creatinina sérica, función renal, glomerulopatía, hipotiroidismo, polimiositis, rabdomiolisis.

\section{Abstract}

Background: In patients with hypothyroidism is possible to find an increased serum creatinine not only due to renal failure but to myopathy in presence of normal renal function. Two patients, each representing 1 type of a proposed classification regarding thyroid dysfunction and abnormal creatinine are presented, in order to communicate this relationship, until now based on isolated reports.

The first individual had an increased serum creatinine with normal renal function due to hypothyroid myopathy (type 1). Rhabdomyolysis related to this myopathy is pointed as a cause of acute renal failure. The 2 nd patient had an increased serum creatinine with abnormal kidney function (type 2), which improved when he became euthyroid after levothyroxine administration.

The diagnosis of hypothyroidism is often missed in patients with altered renal function, if it becomes evident and is properly treated the renal function will improve. As is known hypothyroidism causes hypercholesterolemia, if such patient unknowingly receives statins or gemfibrozil, severe rhabdomyolysis and renal falilure may occur, as reported in the literature. The $3 \mathrm{~d}$ category of the classification is the 
occurrence of nephrotic syndrome in hypothyroid patients who discontinue T4 treatment due to thyroid cancer for further studies or by patient non adherence, developing significant proteinuria few months later. Kidney biopsy lesions vary from membranous glomerulopathy to membranoproliferative glomerulonephritis. These findings will resolve with the administration of levothiroxine, without glucocorticoids. However patients with nephrotic syndrome related to Hashimoto thyroiditis will improve with glucocorticoids.

Conclusion: 1 . TSH must be requested when: a) There is an increased serum creatinine or hypercholesterolemia without a clear cause. b) In patients with rabdomyolysis or acute renal failure with increased CK since it may be secondary to hypothyroid myopathy. 2. Serum creatinine should be requested in hypothyroid patients who discontinue levothyroxine for any reason or are using amiodarone. 3) Proteinuria within the nephrotic range in a hypothyroid patient who has discontinued levothiroxine will have a different response to treatment depending of the cause (if it is autoimmune it will respond to glucorticoids and if is not, will respond to levothyroxine.

Keywords: serum creatinin, renal function, glomerulopathy, hypothyroidism, polymyositis, rabdomyolysis

Recibido: 16 de julio de 2007

Aceptado: 16 de septiembre de 2008

La función renal está muy relacionada con la fisiopatología de la glándula tiroides. En pacientes hipotiroideos se reporta aumento de la filtración glomerular al recibir hormona tiroidea.

En el hipotiroidismo se presentan cambios hemodinámicos renales. En un estudio de 2004, de la función renal realizada con radioisótopos en hipotiroideos, se encontró que el isótopo que mide la función glomerular detectó disminución de la filtración glomerular, mientras que el que valora la función tubular no evidenció alteraciones de esta. ${ }^{1}$

En hipotiroideos, la valoración de la función renal se debe basar en variaciones de los niveles de creatinina sérica $(\mathrm{CrS})$ y del nitrógeno ureico (UN), pruebas más asequibles al médico general, que un aclaramiento de creatinina. Con frecuencia la $\mathrm{CrS}$ puede aumentar en hipotiroideos con función renal normal, como una complicación común de este padecimiento: la miopatía, incluso con rabdomiolisis. Frecuentemente existe alteración renal en esta endocrinopatía y el aumento de la CrS sí traduce disminución de la filtración glomerular. En otros casos puede presentarse daño glomerular corroborado con biopsia renal y proteinuria, en el rango de síndrome nefrótico.

\section{Paciente \#I}

Masculino de 55 años, remitido a nefrología por una $\mathrm{CrS}$ de $2.2 \mathrm{mg} / \mathrm{dL}$ (N: 0.4-1.2 mg/dL). Refería cansancio y edema podálico de varios meses de evolución, sin disnea ni antecedente de hepatopatía o alcoholismo. Negó hematuria. El examen físico mostró un paciente con aspecto abotagado y pálido, pero de mucosas con buena coloración. No tenía disnea, ni ingurgitación yugular. Había matidez en ambas bases pulmonares. El corazón tenía tonos débiles. Había edema podálico duro y reflejos rotulianos pendulares, sugerentes de hipotiroidismo. Los exámenes de laboratorio mostraron nitrógeno ureico en $17 \mathrm{mg} / \mathrm{dL}$ y creatinina sérica $(\mathrm{CrS})$ en $2.2 \mathrm{mg} / \mathrm{dL}$. La glicemia en $91 \mathrm{mg} / \mathrm{dL}$. Na $\mathrm{Na}^{+}$érco 140 $\mathrm{mmol} / \mathrm{L}$., colesterol en $350 \mathrm{mg} / \mathrm{dL}$ y triglicéridos en $604 \mathrm{mg} /$ dL. Las proteínas totales y la relación A/G era normal, proteinuria de 24 horas fue de: $263 \mathrm{mg} / \mathrm{vol}$, volumen urinario $2.92 \mathrm{~L}$ en 24 horas con aclaramiento de creatinina normal. El diagnóstico clínico fue de hipotiroidismo. La primera determinación de TSH estaba en $195 \mathrm{mUI} / \mathrm{mL}$ y el control en $199 \mathrm{mUI} / \mathrm{mL}$, (N: $0.4-4.5 \mathrm{mUI} / \mathrm{mL}$.$) .$

Se inició tratamiento con levotiroxina (LT4) $50 \mu \mathrm{g}$ por 8 días y paulatinamente se aumentó a $100 \mu \mathrm{g}, 150 \mathrm{y}$ $200 \mu \mathrm{g}$; 6 semanas después la creatinina estaba en $1.2 \mathrm{mg} /$ $\mathrm{dL}$; y en $1.1 \mathrm{mg} / \mathrm{dl}$ a los 2 meses. Ha persistido eutiroideo hasta la fecha tomando $150 \mu \mathrm{g}$ por día de LT4.

\section{Paciente \#2}

Diabético de 77 años, con antecedente de insuficiencia cardiaca congestiva (ICC). Consultó por insuficiencia renal de aparición reciente. Antecedente quirúrgico de tiroidectomía y tomaba amiodarona. Se encontraba muy edematoso y previa consulta con su cardiólogo se le suspendió la amiodarona ${ }^{6-7}$ y se le ajustó la dosis de LT4. La proteinuria fue reportada en $119 \mathrm{mg} / \mathrm{vol}$ orina de 24 horas. La TSH era de $46.7 \mathrm{mUI} / \mathrm{ml}$, la creatinina sérica, de $2.04 \mathrm{mg} / \mathrm{dl}$. Tres meses después estaba eutiroideo. $\mathrm{Su}$ peso bajó de $91.5 \mathrm{~kg}$ a 82.6 y la creatinina a $1.2 \mathrm{mg} / \mathrm{dl}$.

\section{Discusión}

La presencia de $\mathrm{CrS}$ elevada con función renal normal en el primer paciente obligó a buscar una explicación. Kreisman ya habia comentado que "la asociación entre hipotiroidismo y una creatinina sérica elevada no está incluida en los libros de texto estándar de medicina interna o subespecialidades hoy, pero debería estar en el futuro". ${ }^{8}$ Suelen mencionarse los cambios hemodinámicos, pero se omiten las alteraciones en el nitrógeno y en la $\mathrm{CrS}$. Estos cambios producen disminución de la filtración glomerular (FG) y del flujo plasmático renal. ${ }^{1}$ Moses relaciona los cambios en el descenso de la FG con una proporción entre la vasoconstricción generalizada y el aumento en la resistencia vascular sistémica. ${ }^{9}$ 
La interrelación entre hipotiroidismo y función renalestructura puede clasificarse en los siguientes 3 tipos: a) CrS elevada en pacientes con filtración glomerular normal; b) CrS elevada en pacientes con disminución de la función renal; c) pacientes con glomerulopatías relacionadas con la suspensión del tratamiento con LT4 ó con cuadros autoinmunes de la tiroides.

\section{CrS elevada en pacientes con filtración glomerular normal}

Son pacientes con hipotiroidismo sintomático no diagnosticado; algunos con rabdomiolisis franca, otros con aumento de creatinina fosfocinasa (CK) y cuadro de polimiositis. ${ }^{10-13} \mathrm{El}$ hipotiroidismo suele ser profundo, como en el primer paciente arriba descrito. Tienen incremento de $\mathrm{CrS}$, por aumento de la generación de esta, relacionados con trastornos musculares típicos del padecimiento. Presentan fatiga, mialgias, rigidez, reflejos lentos, debilidad muscular proximal, dolor a la palpación muscular, marcado aumento de $\mathrm{CK}$ e incremento de la $\mathrm{CrS}$. La rabdomiolisis es frecuente y causa insuficiencia renal aguda, ${ }^{14-15}$ y es preciso su diagnóstico diferencial. ${ }^{16}$ Se debe enfatizar la relación insuficiencia renal aguda / hipotiroidismo, condicionada por rabdomiolisis. ${ }^{17} \mathrm{La} \mathrm{CrS}$ puede elevarse por aumento de generación, inhibición de la secreción renal o por disminución de la función renal.

\section{A) Aumento de la generación de la creatinina}

En 1994 se reportó un paciente de 40 años con aumento progresivo de la creatinina que pasó de $1.6 \mathrm{mg}$ / dl a 1,8 mg/dl (normal 0.4-1.5 mg/dl); 2 meses más tarde, los electrolitos estaban normales y la CK en 2077 U/L. Las pruebas de función tiroidea mostraron un T4 bajo $(2.9 \mathrm{mIU} / \mathrm{ml})$ y la $\mathrm{TSH}$ aumentada $(89 \mathrm{mUI} / \mathrm{mL})$. La función renal medida por 2 métodos diferentes fue normal, con aclaramiento de creatinina de $118 \mathrm{ml} / \mathrm{min}$ y la $\mathrm{FG}$ medida con iodotalamato marcado, estaba en $101 \mathrm{ml} / \mathrm{min}$ $\mathrm{x} 1.73 \mathrm{~m} .^{2}$ Tenía aumento de la excreción de creatinina en orina de 24 hs $(2.72 \mathrm{~g} /$ día ó $35 \mathrm{mg} / \mathrm{kg} /$ día), (N: 20-25mg/ $\mathrm{kg}$ x día). Dos meses después de iniciado el tratamiento con levotiroxina, la $\mathrm{CrS}$ y la $\mathrm{CK}$ disminuyeron. El aumento de creatinina urinaria planteó la posibilidad de un exceso de generación de creatinina. ${ }^{10}$ Puesto que en hipotiroidismo el compromiso muscular es tratable y reversible, a todo paciente con miopatía sin causa definida se le ordenan las pruebas de función tiroidea. ${ }^{11}$

\section{B) Inhibición de la secreción renal de creatinina}

La secreción de creatinina puede ser bloqueada por fármacos catiónicos como cimetidina, trimetoprim $\mathrm{y}$ probenecid. ${ }^{18}$ Estudios recientes muestran que el hOCT2 y no el hOCT1 es el responsable de este transporte en la membrana basolateral de las células del túbulo renal humano. ${ }^{19}$
Tras la deshidratación no enzimática de la creatina muscular, la creatinina es sintetizada en el hígado y transportada activamente al músculo. En el primer paciente, el aumento de creatinina obedeció al incremento de generación de esta por alteración muscular, pues siempre la función renal fue normal y estaba hipotiroideo.

\section{C) Disminución de la función renal}

En estos pacientes se documenta la disminución de la función renal por otros métodos de medición de la filtración glomerular $(\mathrm{FG})^{1}$ como los isotópicos, y además hay aumento de la $\mathrm{CrS} .{ }^{20}$

\section{Aumento de la $\mathrm{CrS}$ por disminución de la función renal en el paciente hipotiroideo}

La disminución de la función renal vinculada con hipotiroidismo se presenta en diversas formas: a) Se han descrito grupos de pacientes con insuficiencia renal crónica e hipotiroidismo, quienes al recibir tratamiento sustitutivo con LT4 mejoran su función renal. ${ }^{1,21-24}$ Montenegro ${ }^{21}$ reportó 41 pacientes hipotiroideos, todos con disminución de la FG, y la CrS, las cuales se normalizaron con tratamiento de reemplazo con LT4. Se detectó más hiponatremia en los casos más severos y de mayor edad. Nakahama ${ }^{22}$ trató 2 pacientes con insuficiencia renal crónica, UN de $76 \mathrm{mg} / \mathrm{dL}$ y creatininas de $4.7 \mathrm{mg} / \mathrm{dl}$ y $3.2 \mathrm{mg} / \mathrm{dL}$, bajando esta a 3.0 $\mathrm{mg} / \mathrm{dl}$ y $2.0 \mathrm{mg} / \mathrm{dl}$, respectivamente, después de 13 semanas de tratamiento con LT4. Estos pacientes tenían también miopatía. En la insuficiencia renal se afecta la función de la hormona tiroidea por alteración de su metabolismo periférico y posible reducción de su contenido tisular. También existe aumento del contenido de yodo de la glándula tiroidea. ${ }^{25}$ Es importante mencionar estos cambios propios de los pacientes con IRC, en quienes a pesar de las bajas T3 y T4, la TSH no se eleva y no presentan signos de hipotiroidismo. ${ }^{25}$ b) Pacientes con insuficiencia renal aguda sobre una crónica. Se reporta en la bibliografía una paciente de 70 años con nefropatía isquémica e hipotiroidismo primario, ${ }^{26}$ cuya función tiroidea había sido normal antes de la exacerbación del fallo renal, pero durante este se encuentra disminuida y con TSH muy alta. Recibió tratamiento con LT4, que produjo rápida mejoría de su función renal. Se sugiere, por lo tanto, solicitar pruebas de función tiroidea a pacientes con deterioro de la función renal sin causa evidente. ${ }^{26}$ Existen también múltiples estudios de hipotiroidismo y nefropatía diabética que permiten afirmar que el hipotiroidismo subclínico aumenta el riesgo de nefropatía diabética. ${ }^{27}$

El diabético con carcinoma papilar de tiroides aumenta la $\mathrm{CrS}$, igual que en los pacientes con cáncer de tiroides y disminución de la función renal en quienes se ha suspendido el tratamiento con T4 para que aumente la TSH y la captación con el fin de que se manifiesten las posibles metástasis. ${ }^{28}$ Además, en los diabéticos tratados con metformina se aumenta la creatinina, medicamento que no 
se debe usar si la $\mathrm{CrS}$ es $>1,5$ en hombres y $1.4 \mathrm{mg} / \mathrm{dl}$ en mujeres, o si el aclaramiento es $<60 \mathrm{~mL} / \mathrm{min} .{ }^{28} \mathrm{En}$ pacientes renales, diabéticos o no, que presenten causa no clara de deterioro de la función renal, debe medirse la TSH. ${ }^{29}$ c) Pacientes trasplantados con hipotiroidismo, en quienes al mejorar la función renal se tuvo que disminuir la dosis de LT4 .30 Un estudio de pacientes trasplantados, con hemodiálisis o diálisis peritoneal ambulatoria crónica, mostró que tenían un tamaño mayor de la glándula tiroides. No existe una relación entre la TSH y el tamaño de la glándula. ${ }^{31}$

En niños con hipotiroidismo congénito ${ }^{32} \mathrm{o}$ adquirido y diferentes grados de disfunción renal, urge el tratamiento temprano con LT4, pues al tratar el hipotiroidismo suele mejorar la nefropatía. ${ }^{3,34,36}$

\section{Glomerulopatías relacionadas con suspensión del tratamiento con LT4 ó con cuadros tiroideos autoinmunes.}

Consiste en la aparición de glomerulopatía al interrumpir el tratamiento; reportado en dos pacientes japonesas hipotiroideas, quienes presentaron a los 6 meses de la suspensión proteinuria, edema, aumento de peso y disfunción renal y cuya biopsia renal mostró en ambas glomerulopatía focal segmentaria. ${ }^{37}$

Estudios de indican muestran diversos tipos de glomerulopatías: fibrilar, membranosa e incluso membrano proliferativa con síndrome nefrótico, que desaparece al recobrar el estado eutiroideo. ${ }^{38}$

Es bastante frecuente la glomerulopatía membranosa asociada con patología tiroidea. ${ }^{39}$ Varios estudios españoles han reportado enfermedad glomerular asociada con enfermedades tiroideas, glomerulopatía membranosa, glomerulonefritis membranoproliferativa ${ }^{40}$ con tiroiditis autoinmune. Estos pacientes tienen la particularidad de que responden a bolos de glucocorticoides, mientras que los anteriores respondían a LT4.

\section{Conclusiones}

En el diagnóstico diferencial de polimiositis siempre debe considerarse la posibilidad de hipotiroidismo. La determinación de TSH debe incluirse como prueba de cribaje en pacientes con fallo renall agudo o crónico, quienes presenten empeoramiento de la función renal por insuficiencia renal aguda sobre una crónica, deben estudiarse por hipotiroidismo con TSH. Los pacientes diabéticos o no que presenten alteración de su función renal, deben también tener una prueba de TSH. En niños con hipotiroidismo congénito o adquirido es preciso buscar alteraciones de la función renal.

En los hipotiroideos que suspenden o disminuyen el tratamiento con hormona tiroidea y presentan edemas, aunque estos pueden ser propios del hipotiroidismo, pueden también ser por síndrome nefrótico, el cual desaparecerá al volver a estar eutiroideos, sin necesidad de administrar glucocorticoides. Algunos pacientes con tiroiditis de Hashimoto pueden presentar glomerulonefritis; si la proteinuria tiene el rango de síndrome nefrótico, responde a glucocorticoides, por tener esta una base autoinmune.

\section{Referencias}

1. Karanikas G, Schutz M, Becherer A, Wiesner K, Dudczak P. Isotopic renal function in severe hypothyroidism and after thyroid hormone replacement therapy. Am J Nephrol 2004; 24:41-45.

2. Del-Rio G, Tapia L, Picazo B, Ruiz JA, Hortas ML, Romero J. Renal failure and acquired hypothyroidism. Pediatric Nephrol 2003; 18:290292.

3. Yeter E, Keles T, Durmaz T, Bozkurt E. Rabdomyolysis due to additive effect of statin therapy and hypothyroidism: a case report. J Med Case Reports 2007; 1:130-133.

4. Barquero J, Rodríguez C, Arrobas I. [Gemfibrozil induced rhabdomyolysis in a patient with undiscovered hypothyrodism]. An Med Interna 2006; 23:504-505.

5. Paydas S, Gokel Y. Different renal pathologies associated with hypothyroidism. Ren. Failure 2002; 24:595-600.

6. Jameson JL, Weetman AP. Trastornos de la glándula tiroides. Efectos de la Amiodarona sobre la función tiroidea. En Harrison Endocrinología. Jameson J, Larry Md.Phd Ed. McGraw-Hill. Interamericana. Madrid. 2006.

7. Iervasi G, Clerico A, Bonini R, Manfredi C. Acute effects of amiodarone administration on thyroid function in patients with cardiac arrthythmia. J Clin Endocrinol Metab 1997; 82:275-280.

8. Kreisman S.H, Hennessey J.V. Consistent reversible elevations of serum creatinine levels in severe hypothyroidism. Arch Intern Med 1999; 159:79-82.

9. Moses MA, Scheinman SJ. The kidney and electrolyte metabolism in hypoth yroidism. In Braverman LE, Utiger RD eds. Werner and Ingbar's The Thyroid 7th ed. Philadelphia. Pa. Lippincott-Raven Press.1996, p: 812-815.

10. Lafayette RA, Costa ME, King AL Increased serum creatinine in the absence of renal failure in profound hypothyroidism. Am J Med 1994; 96:298-299.

11. Khaleeli AA, Edwards RTH. Effect of treatment on skeletal muscle dysfunction in hypothyroidism. Clin Sci 1984; 66: 63-68.

12. Cabili S, Pines A, Kaplinski N, Frankl O. Hypothyroidism masquerading as polymyositis Postgrad Med 1982; 58:545-547.

13. Plotz PH. Not myositis: A series of chance encounters. JAMA 1992; 268:2074- 2077.

14. Birewar S, Oppenheimer M, Zawada ETJr. Hypothyroid acute renal failure. S J D Med 2004; 57;109-110.

15. Jain S, Bhargava K, Sawlane KK, Daga MK. Myoglobinuria and transient acute renal failure in a patient revealing hypothyroidism. J Assoc Physicians India. 1999; 47:444-446.

16. Melli G, Chaudhry V, Cornblath DR. Rabdomyolysis: and evaluation of 475 hospitalized patients. Medicine (Baltimore). 2005; 84:377385.

17. Kar PM, Hirani A, Allen MJ. Acute renal failure in a hypothyroid patient with rhabdomyolysis. Clin Nephrol.2003; 60:428-429.

18. Preuss HG. Organic cationic drugs and renal creatinine secretion. Renal Physiol 1983; 6:103.

19. Urakami Y,Kimura N, Okuda M, Inui K . Creatinine transport by basolateral organic cation transporter hOCT2 in the human kidney. Pharm Res 2004; 21:976-981. 
20. Levey AS. Perrone RD. Madias NE. Serum creatinine and renal function. Ann. Rev. Med 1988; 39:465-490.

21. Montenegro J, González O, Saracho R. Changes in renal function in primary hypothyroidism Am J Kidney Dis 1987; 253: F170-F179.

22. Nakahama H, Sakaguchi K, Horita WW. Treatment of severe hypothyroidism reduced serum creatinine levels in two chronic renal failure patients. Nephron 2001; 88:264-267.

23. Den Hollander JG, Wulkan RW, Mantel MJ, Berghout A. Correlation between severity of thyroid dysfunction and renal function Clin Endocrinol (Oxf) 2005; 62:423-427.

24. Mooraki A, Broumand B. Reversible acute renal failure associated with hypothyroidism: Report of four cases with a brief review of literature. Nephrology 2203; 8:57-60.

25. Lim VS. Thyroid function in patients with chronic renal failure. Am J Kidney Dis. 2001; 38 (Suppl)S80-S84.

26. Makino Y, Fujii T, Kuroda S, Inenaga T et al. Exacerbation of renal failure due to hypothyroidism in a patient with ischemic nephropathy. Nephron 2000; 84:267-269.

27. Chen SS, Wu TE, Jap TS, Wang Hl. Subclinical hypthyroidism is a risk factor for nephropathy and cardiovascular diseases in type 2 diabetic patients. Diabet Med 2007; 24:1336-1344.

28. Bernet VJ Reversible renal insuficiency attributable to thyroid withdrawl in a patient witn type 2 Diabetes mellitus. Endocr Pract 2004; 10:339-344.

29. Lo JC, Chertow GM, Go AS, Hsu CY. Increased prevalence of subclinical and clinical hypothyroidism in persons with chronic kidney disease. Kidney Int 2005; 67:1047-1052.

30. Thomas MC, Mathew TH, Russ GR. Changes in thyroxin requirements in patients with hypothyroidism undergoing renal transplantation Am J Kidney Dis 2002; 39: 354-35.
31. Lebkowska U, Nalysko J, Nysliwiec N. Thyroid function and morphology in kidney transplant recipients, hemollized and peritoneally dialysed patient. Transplant Proc 2003; 35:2945-2948.

32. Asami T, Uchiyama M. Elevated serum creatinine level in infants with congenital hypothyroidism: reflection of decreased renal function? Acta Paediatr 2000; 88:1431-1434.

33. Bald M, Hauffa BP. Wingen AM. Hythyroidism mimicking chronic renal failure in reflux nephropathy. Arch Dis Child 2000; 83:251252.

34. Matoo TK. Hypothyroidism in infants with nephrotic syndrome. Pediatric Nephrol 1994; 8:657-659.

35. Hall C, Batch J, Jones C. Increased serum creatinine associated with severe primary hypothyroidism. J Pediatr Child Care 1996; 32:7374.

36. Al-Fifi S, Girardin C, Sharma A, Rodd C. Moderate renal failure in association with prolonged acquired hypothyroidism in children. Acta Paediatr 1999; 88:715-717.

37. Ogata S, Yorika N, Tangi A. Two cases of hypothyroidism complicated by renal dysfunction. Hiroshima J Med Sci 2000; 49:93-96.

38. Davis RG, Madsen KM, Fregly MJ, Tisher CC. Kidney structure in hypothyroidism. Am J Pathol 1983; 113:41-49.

39. Grcevska L, Polenakovic M, Petrusevka G. Membranous nephropathy associated with thyroid disorders. Nephron 2000; 86:534-535.

40. Valentín M, Bueno B, Gutiérrez E. Membrano proliferative glomerulo nephritis associated with autoimmune thyroidits. Nefrología 2004; 24 Suppl 3:43-48.

\title{
Estado confusional, lesión pulmonar y glúteos que languidecen. Efectos secundarios de procedimientos estéticos
}

\section{(Seconday effects of aesthetic procedures)}

\author{
Julián Peña-Varela, Manuel Fco. Jiménez-Navarrete
}

\section{Resumen:}

Los procedimientos estéticos a cargo de personal no médico son cada vez más frecuentes, muchos no controlados médicamente y promotores de efectos secundarios desde leves a graves. Se presenta el caso de una mujer de 29 años de edad, sin problemas de salud relevantes, quien acudió a un centro de estética para inyectarse silicio orgánico en sus glúteos, con la finalidad de aumentar su volumen, y desarrolló posteriormente un síndrome de embolismo masivo.

Descriptores: embolismo masivo, silicio orgánico

Servicio de Medicina Interna, Hospital México.

Abreviaturas: LCR, líquido cefaloraquídeo; PA, presión arterial; TAC, tomografía axial computarizada

Correspondencia: Manuel Fco Jiménez Navarrete

Email: mickeymfjn@yahoo.com

ISSN 0001-6002/2009/51/1/48-49

Acta Médica Costarricense, (C2009

Colegio de Médicos y Cirujanos

\begin{abstract}
:
Aesthetic procedures performed by non medical personnel are increasing in frequency. Many are not medical controlled and cause side effects from mild to serious. We present the case of a 29-year-old woman, with no relevant health problems, who went to a aesthetics center to have organic silicon injected into her buttocks to increase volume, developing later a massive pulmonary embolism syndrome.
\end{abstract}

Key words: massive embolism syndrome, organic silicium

Recibido: 23 de julio de 2008

Aceptado: 21 de octubre de 2008

En febrero del 2007, se presentó al servicio de urgencias del Hospital México una mujer de 29 años de edad, tabaquista activa ( $1 / 2$ paquete diario), etilista social, con el único 